\title{
Local Information Processing in Adults with High Functioning Autism and Asperger Syndrome: The Usefulness of Neuropsychological Tests and Self-Reports
}

\author{
Annelies A. Spek • Evert M. Scholte • \\ Ina A. Van Berckelaer-Onnes
}

Published online: 23 September 2010

(C) The Author(s) 2010. This article is published with open access at Springerlink.com

\begin{abstract}
Local information processing in 42 adults with high functioning autism, 41 adults with Asperger syndrome and 41 neurotypical adults was examined. Contrary to our expectations, the disorder groups did not outperform the neurotypical group in the neuropsychological measures of local information processing. In line with our hypotheses, the self-reports did show higher levels of local information processing and a stronger tendency to use systemizing strategies in the two disorder groups. Absent and weak correlations were found between the self-reports and the two neuropsychological tasks in the three groups. The neuropsychological tests and the self-reports seem to measure different underlying constructs. The self-reports were most predictive of the presence of an autism spectrum diagnosis.
\end{abstract}

Keywords Local information processing .

High functioning autism · Asperger syndrome .

Embedded figures · Detailed

\section{Introduction}

In order to recommend appropriate guidance and treatment to adults with autism spectrum disorders (ASD), it is important to be aware of the specific impairments and coping mechanisms of these individuals. Knowledge about their strengths and impairments enables the search for

\footnotetext{
A. A. Spek $(\bowtie)$

GGZ Eindhoven, Boschdijk 771, P.O. Box BD6232, 5626 AB

Eindhoven, The Netherlands

e-mail: aa.spek@ggze.nl

E. M. Scholte · I. A. Van Berckelaer-Onnes

Department of Clinical Child and Adolescent Studies, Leiden

University, Leiden, The Netherlands
}

occupations in which they can use their strengths and be restricted only minimally by their impairments. Local information processing has been frequently mentioned as a strength of individuals with ASD (Frith 1989, 2003; Happé and Frith 2006; Jolliffe and Baron-Cohen 1997; Shah and Frith 1993 and others). However, it is yet undetermined whether this also applies to adults with ASD.

Local versus global information processing in children with autism has been a topic of extensive research since 1989 (Frith 1989, 2003; Happé 1996; Jarrold et al. 2000; Morgan et al. 2003; Mottron et al. 2003; Ropar and Mitchell 2001 and others). Whereas global information processing has been characterized as processing information for meaning and gestalt, local information processing can be described as having a bias for featural and detailed information (Happé and Frith 2006). Individuals with autism appear to have a local perceutal bias, since they focus more on the elemental parts of a stimulus and have a strength in detail-focused information processing (Happé 1999).

The local information processing style in individuals with ASD is thought to be underlying areas of talent like memory for exact pitch (Bonnel et al. 2003) and superior visual search (Plaisted et al. 1998). However, the body of research that examined whether and to what extent adults with high functioning autism (HFA) or Asperger syndrome (AS) have a local information processing style is limited and the results of these studies are contradictory (Jolliffe and Baron-Cohen 1997; Kaland et al. 2007; Minshew et al. 2008; Pring et al. 1995; Rumsey and Hamburger 1988). Previous studies used both neuropsychological tests and self-reports to assess local information processing, although it has never been examined whether the two measure a similar underlying construct.

In the present study, local information processing by adults with HFA, AS and a neurotypical adult group will be 
investigated using both neuropsychological tests and selfreport questionnaires. Furthermore, the relationship between the neuropsychological tests and the self-reports will be assessed.

\section{Local Information Processing in Autism}

Frith $(1989,2003)$ was the first to examine local versus global information processing in individuals with autism. In her 'weak central coherence account', she described strengths in local information processing combined with a failure to integrate information into a meaningful whole as characteristic for autism. Throughout the years, the idea of a core deficit in central coherence has been replaced by the suggestion that local, fragmented information processing can be seen as a bias or cognitive style in individuals with autism spectrum disorders (ASD), which can be overcome in tasks that demand global processing (Happé and Frith 2006; Wang et al. 2007). Currently, two prevailing frameworks in local information processing in ASD are the 'Enhanced Perceptual Functioning (EPF) hypothesis' (Mottron et al. 2006), and the 'Empathizing-Systemizing (E-S) account' (Baron-Cohen et al. 2002). The EPF hypothesis states that people with autism display a local bias without evidence of a global deficit (Mottron et al. 2007). According to the E-S account, individuals with autism are more likely to use systemizing strategies. Systemizing can be described as the tendency to analyze information and to construct systems that are lawful. Although the E-S approach is not a local versus global theory of cognition theory per say, it does consider excellent attention to detail as a core characteristic of autism.

\section{Local Information Processing in Adults with ASD}

Studies that examined local information processing specifically in adults are limited and results are contradictory. Although there are no tests developed specifically to examine local information processing, the Embedded figures test (EFT: Witkin et al. 1962) and the Block design subtest of the WAIS III (Wechsler 1997) have been used the most frequently in this account. Research showed that performance on an adapted Block Design task is positively related to autistic traits (Stewart et al. 2009) and generally, superior performance on both tasks is interpreted as a strength in local information processing (Jolliffe and Baron-Cohen 1997; Shah and Frith 1993). However, to our knowledge, only a few studies examined EFT performance in adults with HFA or AS. In one study, superior functioning was found for adult groups with HFA and AS (Jolliffe and Baron-Cohen 1997), while another study in a similar group reported no strengths on this task (Minshew et al. 2008). For the Block Design task, superior performance by adult ASD groups was demonstrated in two studies (Rumsey and Hamburger 1988; Pring et al. 1995). Yet, Kaland et al. (2007) reported no differences between adolescents with AS or HFA and a neurotypical group. In the present study, we use both the EFT and the Block Design task in relatively large adult groups with HFA or AS in order to more thoroughly examine local information processing in these groups.

A recent development in autism research is the use of self-reports to examine cognitive and behavioral features. In order to assess self-perceived local information processing and systemizing tendencies in adults with ASD, the Autism Spectrum Quotient (AQ: Baron-Cohen et al. 2001) and the Systemizing Quotient (SQ: Baron-Cohen et al. 2003) have been developed. Research demonstrated that adults with ASD obtained higher scores for both questionnaires compared to neurotypical adults (Baron-Cohen et al. 2001, 2003; Goldenfield et al. 2005; Hoekstra et al. 2008). Furthermore, AQ performance appears related to SQ performance in an autism spectrum condition group and, in a lesser degree, in a typical group (Wheelwright et al. 2006). Although the use of self-reports in individuals with autism is controversial, adolescents and adults with average verbal ability and a relatively high level of functioning seem able to describe their strengths and weaknesses adequately (Blackshaw et al. 2001; Frith and Happé 1999; Hobson et al. 2006; Spek et al. 2009). However, it has never been formally investigated whether self-report questionnaires and neuropsychological tasks that aim to measure local information processing actually measure similar underlying constructs. Therefore, the present study will examine the relationship between self-reports and neuropsychological tests that that used to measure local information processing.

When examining local information processing, it may be relevant to differentiate between HFA and AS, although it is questionable whether HFA and AS can be differentiated. The validity of AS as a distinct diagnostic entity, separate from other pervasive developmental disorders has not been established or disproved (Eisenmayer et al. 1998; Leekam et al. 2000; Wing 2005; Kamp-Becker et al. 2010). Furthermore, research shows that there are only few qualitative distinctions between HFA and AS; most features appear to be shared or overlapping to some degree (Ghaziuddin and Mountain-Kimchi 2004; Macintosh and Dissanayke 2004; Ozonoff and Griffith 2000). Still, the difference in degree of impairment and in language skills between HFA and AS (Kamp-Becker et al. 2010; Klin et al. 2005; Ozonoff et al. 2000; Spek et al. 2008) convinced us to study the two groups separately, especially since the self-report questionnaires rely on verbal skills.

Two factors that may be relevant to the use of the EFT and the Block Design task are speed of information processing and motor demands. Regarding information 
processing speed: Both tasks make use of a time limit: bonus points can be earned when less time is spent on resolving the items. The impairment in speed of information processing that has been found for children and adults with ASD (Calhoun and Mayes 2005; Mayes and Dickerson 2008; Spek et al. 2008; Yoran-Hegesh et al. 2009) may influence their performance of the EFT and the Block Design task negatively. Motor demands may also influence outcome on these two tasks (Wechsler 1997; Witkin et al. 1962). Therefore, in the present study we used processing speed as a covariate and chose two processing speed tasks that also incorporate motor demands.

\section{Hypotheses of the Present Study}

The present study aimed to examine local information processing in a relatively large group of adults with HFA and AS, using the EFT, the Block Design task, the AQ subscale 'attention to detail' and the SQ. We compared the performance of the HFA and AS groups with an IQ-matched control group of neurotypical adults. In line with the 'enhanced local information processing' theories in autism, we expected that the adult HFA and AS groups would perform better on the EFT and the Block Design task and would receive higher scores on the $\mathrm{AQ}$ and the $\mathrm{SQ}$, compared to the neurotypical group. We investigated the relationships between the neuropsychological instruments (Block Design task and EFT) and the self-reports (AQ and SQ) in the research groups, in order to examine whether and to what extent these instruments measure similar phenomena. Furthermore, since we expect the speed of processing information to influence performance on the EFT and the Block Design task, specifically in the HFA group, we used the processing speed factor scale of the WAIS III as a covariate.

\section{Methods}

Participants and Processes Related to Diagnosis

42 individuals with HFA, 41 individuals with AS and 41 neurotypical adult controls took part in the present study (see Table 1). Participants with genetic conditions or relevant neurodevelopmental conditions (e.g., ADHD, Tourette syndrome) were excluded, as were institutionalized participants and participants with a below average intelligence and verbal ability (scoring 85 or less in full scale intelligence and the verbal comprehension index, as measured by the WAIS-III). Of all participants in the present study, approximately one-third was diagnosed with an autism spectrum disorder in childhood, about one-third had previously received care with an unclear diagnosis and the remaining participants had not been diagnosed until adulthood. In the disorder groups, a standardized diagnostic process was executed, as further described in this paragraph.

The diagnosis of either HFA or AS was established through evaluation of historic and current symptomatology. To gather developmental information, parents were interviewed using the Dutch version of the Autism Diagnostic Interview, Revised version (ADI-R, Lord et al. 1994). When parental information was not available, an older brother or sister was interviewed. In these instances, further information about early childhood was gathered, for example from baby books and early clinical reports. The ADI-R was administered by psychologists who were officially trained in the administration and scoring of this instrument. Research shows that the ADI-R yields excellent reliability and validity when used by trained examiners (Lord et al. 1994). However, since the ADI-R has been validated only for children and adolescents, it is important to use a supplementary instrument in the diagnostic process. The ADI-R is often used in combination with the Autism Diagnostic Observation Schedule (ADOS, Lord et al. 1999). Research shows, however, that the ADOS is under-inclusive in diagnosing mild, verbal adolescents and adults with autistic spectrum disorders (Lord et al. 2000). Therefore, in the present study, a semi-structured interview was administered to all subjects, whereby all ASD criteria of the DSM-IV-TR were assessed by asking the participant standard questions. Following questions were asked until it was clear whether the participant met the specific criterion. This semi-structured interview has been used in previous studies (Spek et al. 2008, 2009). Furthermore, observations

Table 1 Matching variables

\begin{tabular}{llllll}
\hline & HFA & Asperger & Neurotypical & Statistic & $p$ value \\
\hline Gender (M:F) & $42(35: 7)$ & $41(37: 4)$ & $41(30: 11)$ & $\chi^{2}=4.145$ & .13 \\
Handedness (R:L) & $42(39: 3)$ & $41(34: 7)$ & $41(36: 5)$ & $\chi^{2}=1.925$ & .38 \\
Mean age & $37.2(10.8)$ & $41.3(11.5)$ & $39.3(9.7)$ & $F(2,121)=1.498$ & .23 \\
FSIQ* & $108.1(14.3)$ & $112.9(14.8)$ & $114.2(11.5)$ & $F(2,121)=2.311$ \\
VCI** & $109.8(10.8)$ & $110.7(10.7)$ & $112.0(11.6)$ & $F(2,121)=.453$ & .64 \\
\hline
\end{tabular}

* FSIQ Full scale intelligence, measured by the WAIS-III

** VCI Verbal comprehension index, measured by the WAIS-III 
of the participants were gathered systematically during the diagnostic process and in the course of the assessment of the neuropsychological tasks. For instance, observations were made of social and communication skills. These observations were subsequently arranged according to the DSM-IV-TR criteria for ASD (APA 2000). After the diagnostic process described above, the DSM-IV-TR items of ASD were scored, based on the semi-structured interview, the ADI-R and the observations of the participant. Only those participants who met the DSM-IV-TR criteria for the autistic disorder or AS were included in the present study. Because of the controversial nature of the DSM-IV criteria in differentiating between the two disorders (Ghaziuddin et al. 1992; Mayes et al. 2001), additional questions, based on the diagnostic criteria of Gillberg and Gillberg (1989) and ICD-10 (WHO 1993), were asked. When a significant delay in spoken or receptive language or development was present, a diagnosis of AS was excluded, in accordance with the ICD-10 criteria. When there was no delay in development or language, the criteria of Gillberg and Gillberg (1989) were used to diagnose the participants with AS, since these criteria more closely resemble Asperger's own descriptions than the criteria of ICD-10 (Leekam et al. 2000).

\section{Materials Used}

\section{Assessment of Local Information Processing}

To assess local information processing, two neuropsychological tasks and two questionnaires were used, which will be described in the following paragraph. The two neuropsychological tasks have not been developed to measure local information processing specifically, however, they have been used frequently in this respect (Jolliffe and Baron-Cohen 1997; Shah and Frith 1993).

\section{Embedded Figures Test}

In the Embedded Figures Test (Witkin et al. 1962), 12 simple figures have to be traced. These simple figures are embedded in larger, more elaborate designs. The standard procedure as described in the instruction manual was followed. Each complex design was shown for $15 \mathrm{~s}$ and after removal of the complex design, the simple shape card was shown for $10 \mathrm{~s}$. Then the complex design card was shown again and the participant was asked to trace the outline of the shape using a stylus pen. The participants were told that the simple shape card could be re-exposed as many times as they wanted. The average mean time spent to detect each simple figure was used as a dependent variable in the present study. The time the participant needed to trace the figure with the stylus (after having found the figure) was not included in this score, so the total time-score did not reflect any motor demands.

\section{Block Design Task}

The Block Design task is a subtest of the WAIS III (Wechsler 1997). In this task, patterns have to be arranged with blocks that have differently coloured sides. The score obtained reflects whether, and how fast the participant has completed the patterns within a given time limit. In autism research, strengths in performance on the Block Design task have been attributed to strengths in mentally breaking down a whole into its constituent parts (analysis) and then reconstructing the whole from these parts (synthesis). The WAIS-III has been validated for the Dutch population (Wechsler 1997).

\section{Autism Spectrum Quotient}

The AQ is a 50-item self-administered questionnaire that assesses the degree to which an adult recognizes features of the core autistic phenotype (Baron-Cohen et al. 2001). The internal consistency and test-retest reliability are satisfactory (Hoekstra et al. 2008). The AQ subscale 'attention to detail', that was used in the present study, comprises 10 items. Results of a factor-analysis indicated that this subscale can be seen as a separate, valid factor (Hoekstra et al. 2008). In the present study, a Dutch translation of the AQ was used (Ponnet et al. 2001). The 'attention to detail' subscale score was based on the original 4-point Likert scale scores $(1=$ definitely agree, to $4=$ definitely disagree). For six items, the scoring was reversed so that in all items a high score was characteristic for autism. The ten items scores were summed, which resulted in a minimum score of 10 and a maximum score of 40 . The questionnaire was administered as a pen-and-paper task.

\section{Systemizing Quotient}

The Systemizing Quotient (SQ) is a self-report questionnaire, developed to assess systemizing tendencies in adults with normal intelligence (Baron-Cohen et al. 2003). Systemizing can be described as the tendency to analyze information and construct systems that are lawful in order to predict novel situations. The SQ comprises 60 questions: 40 items assess systemizing and 20 are filler items. Individuals score 2 points if they display a systemizing response strongly and 1 point if they display a slightly systemizing response. The possible scores can range from 0 to 80. In the present study, a Dutch translation of the questionnaire was used. 
Assessment of Processing Speed

To assess the speed of information processing, the factor scale 'Processing Speed' of the WAIS III was used (Wechsler 1997). WAIS-III has excellent psychometric properties (Sattler and Ryan 1999) and has been validated for the Dutch population (Wechsler 1997).

The Processing speed factor scale refers to the speed with which cognitive processes are carried out and consists of two paper-and-pencil subtests. In the subtest Digit Symbol-Coding, the participant copies symbols that are paired with numbers. Each symbol is drawn under its corresponding number. The score is determined by the number of symbols correctly drawn. In the subtest Symbol Search, the participant is given rows of symbols and target symbols. They are asked to mark whether or not the target symbols appear in each row.

\section{Procedures}

Recruitment took place from July 2005 to June 2008. The participants of the HFA and the AS groups were recruited from GGZ (Dutch Mental Health Agency) Eindhoven and GGZ Oost-Brabant. They visited one of these mental health agencies for various reasons, for example problems at work and/or marital problems. The neurotypical control subjects were recruited from the general population by adds in local newspapers and by word of mouth. Healthy controls were not included in the present study if they had a history of psychiatric illness or if autism ran in the family. In total, 124 of the 126 possible participants agreed to take part and signed informed consent forms prior to their inclusion in the present study. All participants were tested in a separate quiet room. Breaks in between tasks were given when needed. For the questionnaires, the participants could use as much time as needed. For the Embedded figures test and for the Block Design task, time restrictions were used in accordance with the instruction manuals. The present study was approved by the Ethics Committees of the two participating centers.

\section{Matching Procedure}

The three groups were matched according to age, gender, handedness, full Scale intelligence and verbal abilities. To match for verbal abilities, the WAIS-III factor scale 'Verbal Comprehension Index' (VCI) was used. The subject characteristics for the three groups are presented in Table 1. A Chi-Square test illustrated that the three groups did not differ in gender distribution or handedness. A oneway ANOVA showed that the three groups were comparable in VCI, FSIQ and mean age (see Table 1).

\section{Results}

Differences in EFT Response-Time and Block Design Performance

The mean scores and standard deviations of local information-processing as measured by the EFT and the Block Design task for the HFA group, the AS group and the neurotypical group are presented in Table 2 .

To test the hypothesis of differences in performance on the EFT and the Block Design task between the three groups, two-one-way between-group analyses of variance (ANOVA) were performed, using the diagnosis as the independent variable and the two neuropsychological tests as the dependent variables, respectively. The assumption of homogeneity was met, however, Levene's test (Levene 1960) indicated that the assumption of equality of variance was violated in the analysis. Therefore a more conservative alpha of .025 was set (Tabachnick and Fidell 2007).

For mean response time in the EFT, the results displayed a statistically significant main effect of diagnosis $(F(2,121)=$ $4.76, p=.01$, partial eta squared $=.07)$ with a moderate effect size (Cohen 1988, states that a partial eta squared of more than .06 can be described as a moderate effect size). For the Block Design task, no statistically significant main effect of diagnosis was found $(F(2,121)=.642, p=.53)$. Posthoc Tukey comparisons revealed that the neurotypical group

Table 2 Means and standard deviations for the neuropsychological tests and the questionnaires

\begin{tabular}{llcccc}
\hline & HFA & Asperger & Neurotypicals & Sig & Comparison \\
& $N=42$ & $N=41$ & $21.07(4.79)$ & .000 & AS, HFA $>$ NT \\
AQ subscale & $25.52(6.06)$ & $25.44(5.79)$ & $25.32(9.56)$ & .000 & AS, HFA > NT \\
SQ & $36.00(11.52)$ & $34.24(11.25)$ & $12.93(2.25)$ & .528 & .010 \\
Block design & $12.12(3.63)$ & $12.56(3.67)$ & $25.99(14.08)$ & .005 & AS $>$ NT \\
EFT & $38.71(21.33)$ & $35.65(22.17)$ & $112.24(15.62)$ & AS, NT $>$ HFA \\
Processing speed & $100.19(19.11)$ & $109.44(17.10)$ & & & \\
\hline
\end{tabular}


was significantly faster in the EFT than the HFA group $(p=.01)$. The AS group did not differ in response time from either the neurotypical group or the HFA group.

\section{AQ Detailed Information Processing and Systemizing Tendencies}

To test the hypothesis of differences in self-perceived local information processing and the tendency to systemize, twoone-way between-group analyses of variance (ANOVA) were performed with the diagnosis as the independent variable or factor and the $\mathrm{AQ}$ and the $\mathrm{SQ}$ scores as the dependent variables, respectively. The assumptions of homogeneity and equality of variance were met. Wilks' Lambda was used to measure group differences. For the AQ subscale, the results displayed a statistically significant main effect of diagnosis $(F(2,121)=8.578, p<.01$, partial eta squared $=.12$ ). The effect size can be interpreted as moderate (Cohen 1988). For the SQ, a large and statistically significant main effect of diagnosis was found $(F(2,121)=11.57, p<.01$, partial eta squared $=.16)$. Post-hoc Tukey comparisons revealed that the neurotypical group scored significantly lower on the AQ subscale then the individuals with HFA $(p<.01)$ and the AS group $(p<.01)$. Furthermore, the neurotypical group obtained lower scores on the SQ compared to the HFA $(p<.01)$ and the AS group $(p<.01)$. There were no significant differences between the two disorder groups in the AQ and the SQ. The findings thus support the hypothesis that adults with HFA or AS report higher levels of local information processing and systemizing tendencies compared to the neurotypical adult group (Baron-Cohen et al. 2001, 2003; Goldenfield et al. 2005; Hoekstra et al. 2008).

The Relationship Between the SQ, the AQ Subscale, the EFT the Block Design Task

To investigate whether the self-assessments on the two self-report questionnaires and the performance on the two neuropsychological tasks are related, Pearson productmoment correlation coefficients were calculated. Table 3 presents the results.

Table 3 Correlation coefficients

\begin{tabular}{llllll}
\hline$N=124$ & 1 & 2 & 3 & 4 & 5 \\
\hline 1. AQ subscale & - & & & \\
2. SQ total score & $.58 * *$ & - & & \\
3. Block design & .10 & $.19 *$ & - & \\
$\quad$ task & & & & \\
4. EFT & -.01 & -.07 & $-.63 * *$ & - \\
\hline
\end{tabular}

$* p<.05$

$* * p<.01$
Strong and significant correlations were found between the SQ and the AQ subscale $(r=.58, p<.01)$ and between the EFT and the Block Design task $(r=-.63$, $p<.01)$. The correlation between the SQ and the Block Design task was significant but small $(r=.19, p=.03)$. Other correlations were not significant. To investigate possible group differences, the correlation analysis of the AQ and the SQ was also done within the three groups separately. Strong and significant correlation between the AQ subscale and the SQ existed in all three groups (Autism group $r=.57, p<.01$; Asperger group $r=.41, p<.01$; and Neurotypical group $r=.58, p<.01$ ). This shows that the high correlations hold out in each group separately.

The finding of a strong association between the two neuropsychological tasks and between the two self-report assessments on the one hand and the lack of association between the neuropsychological tasks and self-report local information processing on the other, raises the question whether the two instruments assess a similar underlying construct.

This issue of construct validity was further explored by performing a factor analysis with the two neuropsychological tasks and the two self-report questionnaires as the variables. If all four measures point towards the same underlying construct, this points to the emergence of one factor (Gregory 2007).

Analysis yielded a KMO value above .5, and Barlett's Test of Sphericity was significant at $<.01$, suggesting satisfactory conditions for factor analysis to proceed (Field 2005). In the analysis (method: Principal Components) two components emerged with eigenvalues exceeding 1, explaining 48 and $36 \%$ of the variance, respectively. The Oblimin rotated structure matrix of the two principal components is presented in Table 4.

As Table 4 shows, the EFT and the Block Design task loaded predominantly on component 1 , while the AQ and the SQ assessments loaded predominantly on component 2, with both components being only loosely associated $\left(b_{\text {between factors }}=.11\right)$.

The findings of the analysis indicate that the neuropsychological tasks and the self-reports do not point towards a

Table 4 Principal component analysis: factor loadings (rotated component matrix)

\begin{tabular}{lcc}
\hline Variable & Factor $1^{\mathrm{a}}$ & Factor 2 \\
\hline Embedded figures test & -.907 & \\
Block design task & .894 & \\
SQ total score & & .892 \\
AQ subscale & & .883
\end{tabular}

Rotation method: Oblimin with Kaiser normalization

${ }^{\mathrm{a}} R_{\text {factor } 1-\text { factor } 2}=.11$ 
similar underlying construct, but refer to two different constructs.

Exploration of the Predictive Validity of the SQ, the AQ Subscale, the EFT and the Block Design Task

To examine the ability of the neuropsychological test and self-report questionnaires to predict whether a person belonged to the neurotypical or to one of the diagnostic groups, a discriminant analysis was performed. The Asperger group and the HFA group were merged into one group and a two-group discriminant analysis was performed with the neurotypical group and the merged AS/ HFA group as the dependent variable. This analysis yielded a statistically significant function $\left(\chi^{2}(4)=32.18, p<.01\right)$. Overall the discriminant function successfully predicted outcome for $77 \%$ of the cases, with accurate predictions being made for $77 \%$ of the HFA/Asperger group and $78 \%$ of the neurotypical group. The correlations between the predictor variables and the discriminant function showed that the SQ score $(r=.72)$ and the AQ score $(r=.63)$ are highly relevant in order to determine whether an individual belonged to either the HFA/Asperger group or the neurotypical group, while the EFT $(r=.36)$ and the Block Design task $(r=-.18)$ are less relevant in this respect.

The Influence of Processing Speed on Embedded Figures Test Performance

A one-way between-groups analysis of covariance was conducted to investigate whether the differences in Embedded Figures Test performance between the three groups can be attributed to processing speed differences. After adjusting for the processing speed scores, there was no significant difference between the neurotypical and the HFA group in the Embedded Figures Test $(F(2,120)=$ $2.84, p=.06)$. This suggests that processing speed, as was expected, is an underlying factor of EFT performance in adults with HFA.

\section{Discussion}

The present study aimed to investigate local information processing in adults with HFA or AS and the usefulness of neuropsychological instruments and self-report questionnaires in this respect. We expected to find superior performance on the EFT and the Block Design task in the HFA and the AS group; however, the data of the present study did not support this hypothesis. The three groups did not differ in performance in the Block Design task and the neurotypical group even outperformed the two disorder groups on the EFT. Although the impairment in the EFT in the HFA group can be attributed to their relatively low processing speed group, this does not explain why the expected strengths were not found in the disorder groups.

Although these results are in contrast to previous studies of children and adults with ASD that used the EFT and the Block Design task (Jolliffe and Baron-Cohen 1997; Pring et al. 1995; Rumsey and Hamburger 1988; Shah and Frith 1993), two studies of adolescents and adults with ASD reported similar results (Kaland et al. 2007; Minshew et al. 2008).

As opposed to the results of the neuropsychological tests, the findings of the self-report questionnaires were in line with what we expected to find. The two disorder groups obtained higher scores for both the SQ and the AQ compared to the neurotypical group. Apparently, individuals with HFA or AS perceive themselves as being more detail-oriented and report the use of more systemizing strategies compared to the neurotypical group. These results replicate previous findings for adults with HFA or AS and are in line with the Enhanced Perceptual Functioning (EPF) hypothesis' and the 'Empathizing-Systemizing (E-S) account' (Baron-Cohen et al. 2001, 2003; Hoekstra et al. 2008; Mottron et al. 2006; Wakabayashi et al. 2007).

The contrast between the results of the self-reports and the findings of the neuropsychological tasks is striking. Moreover, the analyses pointed to different underlying constructs. Previous studies reported similar results in other cognitive areas (Veenman 2005). If local information processing is an unitary concept, the results evokes the following explanations: either the neuropsychological tasks or the self reports are valid indicators of local information processing. If, according to the first possibility, the results of the neuropsychological tasks are a valid representation of local information processing, then adults with ASD would not differ from neurotypical adults in this respect. This would indicate that they have 'overgrown' their local information processing bias. It would also suggest that the relatively high level of self-reported local information processing that was found for the disorder groups is not valid. We can think of two possible explanations for this: first, the disorder groups may have adjusted their answers to what, in their opinion, corresponded to their diagnosis. However, this explanation seems unlikely because most of the participants were unaware of their diagnosis until after the neuropsychological testing process took place. Second, it could be argued that a lack of insight influenced the results of the self-report questionnaires for the individuals with ASD. However, this would imply that healthy adults are also unable to determine their level of local information processing, since in this group correlations between the neuropsychological tasks and the self-reports were also low 
or absent. Although it is theoretically possible, it does not seem likely that neurotypical adults with average intellectual capacities have so little insight into their cognitive functions.

According to the second possibility, the self-reports are a valid indicator of local information processing, which implies that the EFT and the Block Design task measure different cognitive features. In favor of this hypothesis is the fact that the performance on two self-report questionnaires appeared to be highly indicative of whether a person belonged to one of the disorder groups or to the neurotypical group, while the neuropsychological tests were less specific in this respect. Furthermore, it is important to note that the EFT and the Block Design task were not developed to measure local information processing. Research indicated that performance in the two tasks can be affected by multiple cognitive features (Happé and Frith 2006; Lezak et al. 2004; Witkin et al. 1962, 1971). For example, right and left hemisphere problems can influence performance on the Block Design task (Lezak et al. 2004). From this perspective, it is possible that the performance by our research groups in the EFT and the Block design subtest was influenced by other cognitive features than local information processing. Following this line of thought, the present data add to a recent discussion about whether cognitive task performance corresponds to performance in the real world, which has been referred to as ecological validity (Chaytor et al. 2006). It appears that a large amount of variation in everyday cognitive and behavioral skills cannot be accounted for in neuropsychological tests. In addition, factors such as compensation strategies, age and environmental characteristics influence test performance and can have a negative impact on ecological validity (Chaytor et al. 2006; Kenworthy et al. 2008). Research in autism showed that various neuropsychological tasks, aiming to examine executive functioning, have questionable ecological validity: emphasis has been put on improving and developing ecological valid tasks in this area (Kenworthy et al. 2008). Based on the results of the present study, parallels can be drawn for local information processing.

Although it seems most plausible that the self-reports provide the most valid representation of local information processing, our proof is only indirect. For instance, selfreport questionnaires are verbal tasks in which comprehension of the various questions is essential. Therefore we need to be careful with conclusions in this respect. It is clear, however, that adults with HFA or AS report to be more detail-prone and more inclined to use systemizing strategies. It is important to take this into account when searching for an optimal educational and work environment where these individuals can use their strengths and abilities.
Although more research on this subject is needed, the results of the present study raise questions about the ability of the EFT and the Block Design task to measure local information processing in adults. There are alternative neuropsychological tasks which can be used to assess local information processing, as for instance a modified Block Design task (Shah and Frith 1993). However, research in high-functioning adults with ASD is limited and there is no information about the ecological validity.

If our results are replicated in future studies in adults, self-reports might be considered first choice for examining local information processing in adults, at least until valid neuropsychological instruments are developed specifically to measure this feature.

With regard to the self-reports, the present study showed that the correlation between the SQ and the AQ subscale is medium to strong in all three groups, which is in line with previous results of Wheelwright et al. (2006). The two questionnaires share a considerable proportion of the variance. Local information processing is apparently related to the use of systemizing strategies. This is in correspondence with the E-S approach, which states that for systemizing, local processing is inevitable because a high systemizing mechanism needs to record each data-point (Baron-Cohen 2006). People with autism appear to use these lawful systems to keep an overview of all the details they are perceiving. This hypothesis supports recent ideas that individuals with autism are able to process information globally when necessary or when instructed to do so (Plaisted et al. 1999). It is interesting that the SQ and AQ subscale are also closely related in the neurotypical group. Systemizing strategies may also be used by healthy individuals as a way of organizing details and predicting change. This indicates that local information processing can be seen as a cognitive style and not as a defect, which is not only present in ASD but also in the general population. The idea of local information processing as a style rather than a deficit lends itself to a continuum approach, which is in line with recent perspectives on autism (Rapin 2005). In this view, individuals with ASD can be placed at the extreme end of the continuum, whereas people with impaired local information processing are placed at the opposite end of the same continuum.

In the present study, we differentiated the individuals with HFA group from those with AS, since research has shown that the degree of impairment in various areas is different in the two groups (Klin et al. 2005). Contrary to our expectations, no differences in the neuropsychological test results or in the self-report measures were found between the HFA and the AS group. It may be possible that, because of the relatively high level of functioning, differences in impairment between individuals with HFA and AS diminish during their lifetime. The results of the 
present study confirm previous studies which stressed the questionable validity of identifying autism and AS as separate disorders (Volkmar and Klin 2005).

\section{Limitations}

In the present study, all participants had at least average verbal ability. It is possible that the verbal ability of the participants have influenced performance on the tasks and questionnaires. Therefore, the results of the present study cannot be generalized to individuals with ASD who are less verbally capable. Furthermore, the relatively late diagnosis of a proportion of the participants characterizes our research group. A relatively late diagnosis has been hypothesized to be related to milder symptoms (Vermeulen 2002). However, all the individuals in the disorder groups matched criteria for HFA or AS and individuals with relatively mild symptoms were not included in the present study because they were, generally, diagnosed with PDDNOS.

The present study used two self-report questionnaires to assess local information processing and systemizing tendencies. An adequate understanding and interpretation of the questions used in the questionnaires relies on semantic capacities. Although the two disorder groups were carefully selected and all participants had at least average verbal abilities, deficiencies in semantic processing which characterize individuals with ASD may have influenced performance in the two questionnaires.

Acknowledgments The present article was submitted as part of the first author's PhD Thesis at the University of Leiden. The second and third authors are members of the Department of Clinical Child and Adolescent Studies at Leiden University. The article was written while the first author was employed by the Mental Health Center Eindhoven (GGZ Eindhoven). Thanks go to colleagues in Eindhoven and Oss who helped with the study. We are grateful to all of the participants in the present study.

Open Access This article is distributed under the terms of the Creative Commons Attribution Noncommercial License which permits any noncommercial use, distribution, and reproduction in any medium, provided the original author(s) and source are credited.

\section{References}

American Psychiatric Association. (2000). Diagnostic and statistical manual of mental disorders (4th ed.), text revision. Washington, DC: American Psychiatric Association.

Baron-Cohen, S. (2006). The hyper-systemizing, assortative mating theory of autism. Progress in Neuro-Psychopharmacology and Biological Psychiatry, 30(5), 865-872.

Baron-Cohen, S., Richler, J., Bisarya, D., Gurunathan, N., \& Wheelwright, S. (2003). The systemizing quotient: An investigation of adults with Asperger syndrome or high-functioning autism, and normal sex differences. Philosophical Transactions of the Royal Society, Series B, Special issue on "Autism: Mind and Brain", 358, 174-361.

Baron-Cohen, S., Wheelwright, S., Griffin, R., Lawson, J., \& Hill, J. (2002). The exact mind: Empathizing and systemizing in autism spectrum conditions. In U. Goswami (Ed.), Handbook of cognitive development (pp. 491-508). Malden, M.A., USA: Blackwell Publishers.

Baron-Cohen, S., Wheelwright, S., Skinner, R., Martin, J., \& Clubley, E. (2001). The Autism-spectrum quotient (AQ): Evidence from Asperger syndrome/high functioning Autism, males and females, scientists and mathematicians. Journal of Autism and Developmental Disorders, 31, 5-17.

Blackshaw, A. J., Kinderman, P., Hare, D. J., \& Hatton, C. (2001). Theory of mind, causal attribution and paranoia in Asperger syndrome. Autism, 5(2), 147-163.

Bonnel, A., Mottron, L., Peretz, I., Trudel, M., Gallun, E., \& Bonnel, A. M. (2003). Enhanced pitch sensitivity in individuals with autism: A signal detection analysis. Journal of Cognitive Neuroscience, 15, 226-235.

Calhoun, S. L., \& Mayes, S. D. (2005). Processing speed in children with clinical disorders. Psychology in the Schools, 42(4), 333-343.

Chaytor, N., Schimitter-Edgecombe, M., \& Burr, R. (2006). Improving the ecological validity of executive functioning assessment. Archives of Clinical Neuropsychology, 21, 217-227.

Cohen, J. (1988). Statistical power analysis for the behavioural sciences. Hillsdale, NJ: Erlbaum.

Eisenmayer, R., Prior, M., Leekam, S., Wing, L., Ong, B., Gould, J., et al. (1998). Delayed language onset as a predictor of clinical symptoms in pervasive developmental disorders. Journal of Autism and Developmental Disorders, 28(6), 527-533.

Field, A. (2005). Discovering statistics using SPSS. London: Sage.

Frith, U. (1989). Explaining the Enigma. Oxford: Blackwell Publishing.

Frith, U. (2003). Autism. Explaining the Enigma. Oxford: Blackwell Publishing.

Frith, U., \& Happé, F. (1999). Theory of mind and self-consciousness: What is it like to be autistic? Mind and Language, 14(1), $1-22$.

Ghaziuddin, M., \& Mountain-Kimchi, K. (2004). Defining the intellectual profile of Asperger syndrome: Comparison with high-functioning autism. Journal of Autism and Developmental Disorders, 34, 279-284.

Ghaziuddin, M., Tsai, L. Y., \& Ghaziuddin, N. (1992). A comparison of the diagnostic criteria for Asperger syndrome. Journal of Autism and Developmental Syndromes, 22, 634-639.

Gillberg, I. C., \& Gillberg, C. (1989). Asperger syndrome-some epidemiological considerations: A research note. Journal of Child Psychology and Psychiatry, 30, 631-638.

Goldenfield, N., Baron-Cohen, S., \& Wheelwright, S. (2005). Empathizing and systemizing in males, females and autism. Clinical Neuropsychiatry, 2(6), 338-345.

Gregory, R. J. (2007). Psychological testing. History, principles and applications. London: Pearson Education, Inc.

Happé, F. (1996). Studying weak central coherence at low levels: Children with autism do not succumb to visual illusions, a research note. Journal of Child Psychology and Psychiatry, 37, 873-877.

Happé, F. (1999). Autism: Cognitive deficit or cognitive style? Trends in Cognitive Sciences, 3(6), 216-222.

Happé, F., \& Frith, U. (2006). The weak central coherence account: Detail-focused cognitive style in autism spectrum disorders. Journal of Autism and Developmental Disorders, 36(1), 5-25.

Hobson, R. P., Chidambi, G., Lee, N., \& Meyer, J. (2006). Foundations for self-awareness: An exploration through autism. New York: Wiley-Blackwell. 
Hoekstra, R. A., Bartels, M., Cath, D. C., \& Boomsma, D. I. (2008). Factor structure, reliability and criterion validity of the Autismspectrum Quotient (AQ): a study in Dutch population and patient groups. Journal of Autism and Developmental Disorders, 38(8), $1555-1566$.

Jarrold, C., Butler, D. W., Cottington, E. M., \& Jimenez, F. (2000). Linking theory of mind and central coherence bias in autism and in the general population. Developmental Psychology, 36, $126-138$.

Jolliffe, T., \& Baron-Cohen, S. (1997). Are people with autism and Asperger syndrome faster than normal on the embedded figures test? Journal of Child Psychology and Psychiatry, 38, 527-534.

Kaland, N., Mortensen, E. L., \& Smith, L. (2007). Disembedding performance in children and adolescents with Asperger syndrome or high-functioning autism. Autism, 11(1), 81-92.

Kamp-Becker, I., Smidt, J., Ghahreman, M., Heinzel-Gutenbrunner, M., Becker, K., \& Remschimidt, H. (2010). Categorical and cimensional structure of autism spectrum disorders: The nosologic validity of Asperger syndrome. Journal of Autism and Developmental Disorders, 28, 457-463.

Kenworthy, L., Yerys, B. E., Gutermuth, A. L., \& Wallace, G. L. (2008). Understanding executive control in autism spectrum disorders in the lab and in the real world. Neuropsychology Review, 18, 320-338.

Klin, A., McPartland, J., \& Volkmar, F. R. (2005). Asperger syndrome. In F. R. Volkmar, P. Rhea, A. Klin, \& D. Cohen (Eds.), Handbook of Autism and pervasive developmental disorders (pp. 88-125). New Jersey: Wiley.

Leekam, S., Libby, S., Wing, L., Gould, J., \& Gillberg, C. (2000). Comparison of ICD-10 and Gillberg's criteria for Asperger syndrome. Autism, 4, 11-28.

Levene, H. (1960). Robust tests for equality of variances. In I. Olkin, S. G. Ghurye, W. Hoeffding, W. G. Madow, \& H. B. Mann (Eds.), Contributions to probability and statistics: Essays in honor of harold hotelling (pp. 278-292). Menlo Park, CA: Stanford University Press.

Lezak, M. D., Howieson, D. B., \& Loring, D. W. (2004). Neuropsychological assessment (4th ed.). Oxford: University Press.

Lord, C., Risi, S., Lambrecht, L., Cook, E. H., Leventhal, B. L., DiLavore, P. C., et al. (2000). The Autism diagnostic observation schedule-generic: A standard measure of social and communication deficits associated with the spectrum of autism. Journal of Autism and Developmental Disorders, 30(3), 205-223.

Lord, C., Rutter, M. L., DiLavore, P. C., \& Risi, S. (1999). Autism diagnostic observation schedule. Los Angeles: Western Psychological Services.

Lord, C., Rutter, M., \& Le Couteur, A. (1994). Autism diagnostic interview-revised: A revised version of a diagnostic interview for caregivers of individuals with possible pervasive developmental disorders. Journal of Autism and Developmental Disorders, 24, 659-685.

Macintosh. K., \& Dissanayake, C. (2004). Annotation: The similarities and differences between autistic disorder and Asperger's disorder: A review of the empirical evidence. Journal of Child Psychology and Psychiatry, 45, 421-434.

Mayes, S. D., Calhoun, S. L., \& Crites, D. L. (2001). Does DSM-IV Asperger's disorder exist? Behavioral Science, 29(3), 263-271.

Mayes, S. D., \& Dickerson, S. (2008). WISC-IV and WIAT-II in children with high-functioning autism. Journal of Autism and Developmental Disorders, 3, 428-439.

Minshew, N. J., Williams, D. L, \& Gastgeb, H. (2008, Mai). Inferior performance on Embedded figures tasks by high functioning children and adults with autism consistent with reduced local connectivity \& slower search strategy. In Poster session presented at the international meeting for autism research, London.
Morgan, B., Maybery, M., \& Durkin, K. (2003). Weak central coherence, poor joint attention, and low verbal ability: Independent deficits in early autism. Developmental Psychology, 39, 646-656.

Mottron, L., Burack, J. A., Iarocci, G., Belleville, S., \& Enns, J. T. (2003). Locally oriented perception with intact global processing among adolescents with high-functioning autism: Evidence from multiple paradigms. Journal of Child Psychology and Psychiatry, 44, 904-913.

Mottron, L., Dawson, M., Bertone, A., \& Wang, L. (2007). Cognitive versatility in autism cannot be reduced to a deficit. Cognitive Neuropsychology, 24(5), 578-580.

Mottron, L., Dawson, M., Soulieres, I., Hubert, B., \& Burack, J. (2006). Enhanced perceptual functioning in autism: An update, and eight principles of autistic perception. Journal of Autism and Developmental Disorders, 36, 27-43.

Ozonoff, S., \& Griffith, E. M. (2000). Neuropsychological function and the external validity of Asperger syndrome. In A. Klin, F. R. Volkmar, \& S. S. Sparrow (Eds.), Asperger syndrome (pp. 72-96). New York: Guilford.

Ozonoff, S., South, M., \& Miller, J. N. (2000). DSM-IV-defined Asperger syndrome: Cognitive, behavioral and early history differentiation from high-functioning autism. Autism, 44(1), $29-46$.

Plaisted, K., O'Riordan, M., \& Baron-Cohen, S. (1998). Enhanced visual search for a conjunctive target in autism: A research note. Journal of Child Psychology and Psychiatry, 39, 777-783.

Plaisted, K., Swettenham, J., \& Rees, L. (1999). Children with autism show local precedence in a divided attention task and global precedence in a selective attention task. Journal of Child Psychology and Psychiatry, 40, 733-742.

Ponnet, K., Roeyers, H., \& Buysse, A. (2001). Het Autismespectrum quotient [The Autism-spectrum quotient]. (Baron-Cohen, Wheelwright, Skinner, Martin \& Clubley, 2001), unpublished translation. Ghent: Ghent University.

Pring, L., Hermelin, B., \& Heavey, L. (1995). Savants, segments, art and autism. Journal of Child Psychology and Psychiatry, 36, $1065-1076$.

Rapin, I. (2005). Autism: Where we have been, where we are going? In F. R. Volkmar, P. Rhea, A. Klin, \& D. Cohen (Eds.), Handbook of Autism and pervasive developmental disorders (pp. 1304-1317). New Jersey: Wiley.

Ropar, D., \& Mitchell, P. (2001). Susceptibility to illusions and performance on visuospatial tasks in individuals with autism. Journal of Child Psychology and Psychiatry, 42, 1283-1293.

Rumsey, J. M., \& Hamburger, S. D. (1988). Neuropsychological findings in high-functioning men with infantile autism, residual state. Journal of Clinical and Experimental Neuropsychology, 10(2), 201-221.

Sattler, J. M., \& Ryan, J. J. (1999). Assessment of children, revised: WAIS-III supplement. LeMesa, CA: Jerome Sattler Publishing.

Shah, A., \& Frith, U. (1993). Why do autistic individuals show superior performance on the Block design task? Journal of Child Psychology and Psychiatry, 34, 1351-1364.

Spek, A. A., Scholte, E. M., \& Van Berckelaer-Onnes, I. A. (2008). The use of WAIS-III in adults with HFA and Asperger syndrome. Journal of Autism and Developmental Disorders, 38(4), 782-787.

Spek, A. A., Scholte, E. M., \& van Berckelaer-Onnes, I. A. (2009). Theory of mind in adults with HFA and Asperger syndrome. Journal of Autism and Developmental Disorders, 40, 280-289.

Stewart, M. E., Watson, J., Allcock, A. J., \& Yaqoob, T. (2009). Autistic traits predict performance on the block design task. Autism, 13, 133-142.

Tabachnick, B. G., \& Fidell, L. S. (2007). Using multivariate statistics (5th ed.). Boston: Allyn and Bacon. 
Veenman, M. V. J. (2005). The assessment of metacognitive skills: What can be learned from multi-method designs? In C. Artelt \& B. Moschner (Eds.), Lernstrategien und Metakognition; Implikationen für Forschung und Praxis (pp. 77-99). Münster: Waxmann.

Vermeulen, P. (2002). Beter vroeg dan laat en beter laat dan nooit (Better early than late but better late than never). Berchem: Uitgeverij EPO.

Volkmar, F. R., \& Klin, A. (2005). Issues in the classificaton of autism and related conditions. In F. R. Volkmar, R. Paul, A. Klin, \& D. Cohen (Eds.), Handbook of Autism and pervasive developmental disorders (pp. 606-627). New Jersey: Wiley.

Wakabayashi, A., Baron-Cohen, S., Uchiyama, T., Yoshida, Y., Kuroda, M., \& Wheelwright, S. (2007). Empathizing and systemizing in adults with and without autism spectrum conditions: cross-cultural stability. Journal of Autism and Developmental Disorders, 37(10), 1823-1832.

Wang, L., Mottron, L., Peng, D., Berthiaume, C., \& Dawson, M. (2007). Local bias and local- to-global interference without global deficit: A robust finding in autism under various conditions of attention, exposure time, and visual angle. Cognitive Neuropsychology, 24, 550-574.
Wechsler, D. (1997). WAIS-III administration and scoring manual. San Antonio, TX: The Psychological Corporation.

Wheelwright, S., Baron-Cohen, S., Goldenfeld, N., Delaney, J., Fine, D., Smith, R., et al. (2006). Predicting Autism spectrum quotient (AQ) from the systemizing quotient-revised (SQ-R) and empathy quotient (EQ). Brain Research, 1079, 47-56.

Wing, L. (2005). Reflections on opening Pandora's box. Journal of Autism and Developmental Disorders, 35(2), 197-203.

Witkin, H. A., Dyck, R. B., Faterson, H. F., Goodenough, D. G., \& Karp, S. A. (1962). Personality through perception. New York: Harper \& Row.

Witkin, H. A., Oltman, P. K., Raskin, E., \& Karp, S. (1971). Manual for the embedded figures test. Palo Alto, CA: Consulting Psychologists Press.

World Health Organization. (1993). The ICD-10 classification of mental and behavioral disorders. Geneva: WHO.

Yoran-Hegesh, R., Kertzman, S., Vishne, T., Weizman, A., \& Kotler, M. (2009). Neuropsychological mechanisms of digit symbol substitution test impairment in Asperger disorder. Psychiatry Research, 166, 35-45. 